\title{
PEPTIDE ANTIBIOTIC K-582 PRODUCTION IN RELATION TO AMINO ACID METABOLISM IN METARRHIZIUM ANISOPLIAE
}

\author{
Mikiko Ito-Kagawa, Yasuo Koyama and Shigeji Kondo ${ }^{\dagger}$ \\ Kayaku Antibiotics Research Laboratory Ltd., \\ Funado 2-8-16, Itabashi-ku, Tokyo, Japan \\ ${ }^{+}$Sendai Municipal Institute of Public Health, \\ Sendai, Japan \\ (Received for publication December 1, 1983)
}

\begin{abstract}
Formation of the basic antibiotic, K-582 was stimulated by supplying Metarrhizium anisopliae U-47 with several amino acids present in its structure. The addition of L-arginine to the basal medium resulted in the almost exclusive formation of K-582 B, while L-lysine increased K-582 A formation. Some carbon sources were observed to have effects similar to those obtained with the above mentioned amino acids.

Furthermore, when L-arginine was added in excess to the basal medium, free $\gamma$-hydroxyarginine, which is a major constituent of the antibiotic, accumulated extra- and intra-cellularly. Free $\gamma$-hydroxyarginine isolated from the culture broth of this microorganism was the threo-L-isomer.

K-582 formation was repressed by glycerol, which exerted catabolite repression of $\gamma$ hydroxyarginine synthesis.
\end{abstract}

Imperfect fungus, Metarrhizium anisopliae (Metsch) Sorok var. anisopliae, produces the basic peptide antibiotics, K-582 A and B, which are excreted into the medium; those are effective against yeasts and certain viruses. ${ }^{1)}$ Their chemical structures were determined ${ }^{2)}$ : K-582 A is Arg-Hyarg-Orn-Thr-OrnLys-Tyr; and K-582 B is Arg-Hyarg-Orn-Thr-Orn-Hyarg-Tyr*. $\gamma$-Hydroxyarginine present in K-582 A and B, was isolated from a microbe for the first time, but has been known to be present in the sea cucumber, Polycheira rufescens ${ }^{3)}$, the sea anemone, Anthopleura japonica Verrill $^{4)}$, and the seeds of 17 species of the genus Vicia $^{5)}$. Recently, MizUSAKI and MAKIsUmi ${ }^{6)}$ synthesized chemically four stereoisomers of Hyarg via histidine.

On the other hand, ITO-KAGAWA, one of the authors reported that in biogenesis of another peptide antibiotic, colistin, by Bacillus colistinus Koyama, yields increased markedly when amino acids of the aspartate family (isoleucine, threonine and $\alpha, \gamma$-diaminobutyric acid) were added. ${ }^{7)}$ Specifically, $\alpha, \gamma$ diaminobutyric acid, a major constituent of the colistin molecule, stimulated colistin biosynthesis, and considerably inhibited the biosynthesis of cellular protein. ${ }^{8)}$

The present paper deals with the biogenesis of K-582 in relation to amino acid metabolism and, particularly, the role of Hyarg in the synthesis of K-582. In addition, free Hyarg, which accumulated extra- and intra-cellularly, was isolated and its chemical structure was elucidated.

\section{Methods}

Microorganism

Metarrhizium anisopliae U-47, a mutant producing high yields of K-582 and Hyarg and derived from Metarrhizium anisopliae (Metsch) Sorok var. anisopliae strain No. 582M was used throughout this

* Hyarg: $\gamma$-Hydroxyarginine. 
study. Pullularia pullulans 9173 was used as the test organism for the microbiological determination of K-582.

Media and Cultivation

M. anisopliae U-47 was maintained on slants of $1 \%$ Polypeptone potato agar at $4{ }^{\circ} \mathrm{C}$. The basal medium for fermentation of K-582 was Czapek-peptone medium which contained glucose $3.0 \%$, Polypeptone $1.0 \%, \mathrm{NaNO}_{3} 0.2 \%, \mathrm{KH}_{2} \mathrm{PO}_{4} 0.1 \%, \mathrm{KCl} 0.05 \%, \mathrm{MgSO}_{4} \cdot 7 \mathrm{H}_{2} \mathrm{O} 0.05 \%$ and $\mathrm{FeSO}_{4} 0.001 \%$ (pH 5.4, adjusted) as described previously by Kondo et al. ${ }^{1)}$ Microbiological assay medium contained peptone $1 \%$, meat extract $1 \%$, glucose $0.25 \%$ and $\mathrm{NaCl} 0.5 \%$ (pH 5.8, adjusted).

A loopful of M. anisopliae U-47 from a slant culture was inoculated into a 1-liter Erlenmeyer flask containing $300 \mathrm{ml}$ of the basal medium, the inoculated flask was incubated at $30^{\circ} \mathrm{C}$ for 4 days on a rotary shaker to give the seed culture. An aliquot $(3.0 \mathrm{ml})$ of the seed culture was transferred into a 1-liter Erlenmeyer flask containing $300 \mathrm{ml}$ of the desired medium and incubated as above. At time intervals, samples were taken for $\mathrm{pH}$, growth and antibiotic determination.

Assay Methods

Growth was expressed as $\mathrm{mg}$ of dried cell weight per $\mathrm{ml}$ of culture broth. One $\mathrm{ml}$ of the culture broth was filtered on Toyo Roshi No. 2 filter paper and washed twice with distilled water. The washed mycelium was transferred to a weighing bottle and then dried at 85 to $90^{\circ} \mathrm{C}$ until constant weight.

The intracellular amino acids were extracted with boiling $80 \%$ ethanol from the washed mycelium according to the method of SCHMIT and BRODY ${ }^{8)}$. Amino acid analysis was done with a Jeol automatic amino acid analyzer, model JLC-6AS.

The action of L-amino acid oxidase on $\gamma$-hydroxyarginine was measured by the method of WELLNER and LichtenBurG ${ }^{10)}$. The action of arginase was also assayed by determining the amount of urea formed from the amino acid. The amount of urea was estimated enzymatically using Urea NB-Test "Wako".

Analysis of K-582 A and B

Total activity of K-582 A and B was assayed microbiologically with a paper disk method using $P$. pullulans 9173 as test organism.

The relative amounts of K-582 A and B in the broth filtrate were determined by HPLC (Waters Associates Model $6000 \mathrm{~A})$. Samples were run on a column $(300 \mathrm{~mm} \times 4.6 \mathrm{~mm})$ of microBondapak $\mathrm{C}_{18}$ (Waters Associates, U.S.A.) at room temperature and a flow rate of $2 \mathrm{ml} / \mathrm{minute}$. The eluent consisted of $1 \%$ ethanolamine adjusted to $\mathrm{pH} 3.8$ with phosphoric acid. The absorption peaks were monitored at $280 \mathrm{~nm}$ using Waters Associates Model 440 absorbance detector. Under these conditions, the retention time for K-582 A was about 8 minutes and for K-582 B 11 minutes.

Physico-chemical Measurements

Melting point was determined with a Yanagimoto micromelting point apparatus. Optical rotation was measured in a $0.5 \mathrm{M} \mathrm{NaOH}$ solution using a Jasco DIP-181 automatic polarimeter. The IR spectrum was measured in $\mathrm{KBr}$ tablet using a Jasco IRA-1 spectrometer. The ${ }^{18} \mathrm{C}$ NMR spectra were measured in a Jeol PS-100 ${ }^{13} \mathrm{C}$ NMR spectrometer, with $\mathrm{D}_{2} \mathrm{O}$ as a solvent using dioxane as the internal standard. Chemical shifts were expressed in parts per million (ppm) relative to internal dioxane (67.4 ppm).

\section{Results}

\section{Growth and K-582 Production}

Fig. 1 shows the course of K-582 production by M. anisopliae U-47, the growth and the changes to the medium during fermentation. During the first 3 days of cultivation growth was rapid; glucose was almost exhausted on day 4, after the exponential phase. K-582 production began at the end of the exponential growth phase (day 3 ) and increased markedly until day 5. The relation between growth and K-582 production showed a typical trophophase-idiophase pattern. 
Fig. 1. Time course of K-582 production by $M$. anisopliae $\mathrm{U}-47$ grown in the basal medium.
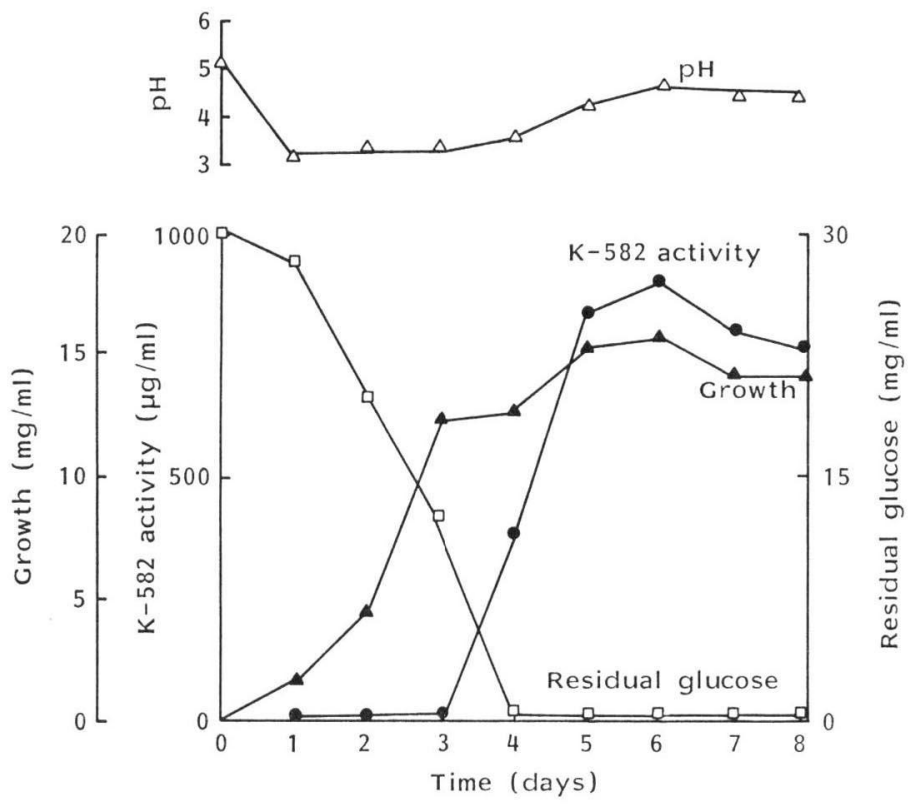

Effect of the Carbon Source on K-582 Production

Several carbohydrates were investigated as carbon sources for K-582 production. Glucose, fructose, sucrose, sorbitol and glycerol were added singly to the basal medium. As shown in Table 1, fructose and sucrose were superior to glucose for K-582 production and favored K-582 A ( 55 and $60 \%$ of the mixture) over K-582 B. Glycerol was excellent for growth of $M$. anisopliae U-47, but completely inhibited K582 production. This suggests carbon catabolite repression of K-582 production in M. anisopliae U-47.

Effect of Amino Acids on K-582 Production

The effect of constituent amino acids, arginine, ornithine, threonine, lysine and tyrosine (not Hyarg) on K-582 production was examined. Each amino acid was added singly at $0.5 \%(\mathrm{w} / \mathrm{v})$ to the basal medium. Arginine, lysine and tyrosine added singly to the basal medium, increased K-582 activity by 20 to $30 \%$ over the basal medium used as a control (Table 2).

The ratio of K-582 A and B produced in the basal medium was 43:57. When L-arginine was

Table 1. Effect of the carbon source ${ }^{\mathrm{a}}$ on growth, $\mathrm{K}-582$ production and $\mathrm{A}$ : $\mathrm{B}$ ratio.

\begin{tabular}{lccc}
\hline $\begin{array}{c}\text { Carbon source } \\
(3 \%)\end{array}$ & $\begin{array}{c}\text { Growth }^{\mathrm{b}} \\
\left(\mathrm{mg} / \mathrm{ml}^{\circ}\right)\end{array}$ & $\begin{array}{c}\mathrm{K}-582^{\mathrm{b}} \\
(\mu \mathrm{g} / \mathrm{ml})\end{array}$ & $\begin{array}{c}\mathrm{K}-582 \\
\mathrm{~A}: \mathrm{B}\end{array}$ \\
\hline Glucose & 14.50 & 1,500 & $44: 56$ \\
Fructose & 16.25 & 1,900 & $60: 40$ \\
Sorbitol & $-{ }^{\mathrm{c}}$ & 1,300 & - \\
Sucrose & - & 2,000 & $55: 45$ \\
Glycerol & 17.40 & 0 & - \\
\hline
\end{tabular}

a Carbohydrate added singly.

b Measured on day 5 .

c Not determined.
Table 2. Effect of amino acids on K-582 production.

\begin{tabular}{lrr}
\hline $\begin{array}{c}\text { Amino acid } \\
\text { added }\end{array}$ & $\begin{array}{c}\mathrm{K}-582 \\
(\mu \mathrm{g} / \mathrm{ml})\end{array}$ & $\begin{array}{c}\mathrm{K}-582 \\
\mathrm{~A}: \mathrm{B}\end{array}$ \\
\hline None & 1,500 & $43: 57$ \\
Arginine & 1,900 & $5: 95$ \\
Lysine & 1,900 & $60: 40$ \\
Ornithine & 1,700 & $37: 63$ \\
Tyrosine & 1,800 & $33: 67$ \\
Threonine & 1,700 & $39: 61$ \\
\hline
\end{tabular}

Each amino acid was added at a concentration of $5 \mathrm{mg} / \mathrm{ml}$ to the basal medium. Cultural conditions and measurements of K-582 activity were carried out as described in Methods. 
Table 3. Intracellular amino acid pools during K-582 production.

\begin{tabular}{|c|c|c|c|c|c|c|}
\hline \multirow{3}{*}{ Amino acid } & \multicolumn{6}{|c|}{ Amino acids ${ }^{\mathrm{a}}$ in mycelia grown on } \\
\hline & \multicolumn{2}{|c|}{ Glycerol $^{1)}$} & \multicolumn{2}{|c|}{ Glucose $^{2)}$} & \multicolumn{2}{|c|}{ Glucose $+\operatorname{arginine}^{3}$} \\
\hline & 4 days & 5 days & 4 days & 5 days & 4 days & 5 days \\
\hline Aspartic acid & 0.514 & 0.559 & 0.502 & 0.497 & 0.297 & 0.247 \\
\hline Threonine & 0.536 & 0.499 & 0.786 & 0.822 & 0.378 & 0.309 \\
\hline Serine & 0.507 & 0.502 & 0.504 & 0.61 & 0.342 & 0.266 \\
\hline Glutamic acid & 1.418 & 1.509 & 1.223 & 1.49 & 0.749 & 0.762 \\
\hline Glycine & trace & trace & 0.117 & 0.096 & 0.076 & trace \\
\hline Alanine & 0.313 & 0.184 & 0.417 & 0.311 & 0.195 & 0.181 \\
\hline Tyrosine & 0.201 & 0.238 & trace & 0.245 & trace & 0.249 \\
\hline Lysine & 0.498 & 0.827 & 0.625 & 1.403 & 0.055 & 0.219 \\
\hline Histidine & 0.234 & 0.261 & 0.366 & 0.489 & 0.079 & 0.201 \\
\hline Arginine & 0.469 & 0.363 & 0.169 & 0.209 & 1.383 & 2.378 \\
\hline Ornithine & 0.267 & 0.214 & 0.743 & 0.421 & 0.180 & 0.073 \\
\hline Hyarg & 0 & 0 & 0.095 & 0.209 & 0.241 & 0.404 \\
\hline $\begin{array}{l}\text { Maximal potency of } \\
\mathrm{K}-582(\mu \mathrm{g} / \mathrm{ml})\end{array}$ & \multicolumn{2}{|c|}{0} & \multicolumn{2}{|c|}{1,500} & \multicolumn{2}{|c|}{1,900} \\
\hline Mol $\%$ of K-582 A and B & \multicolumn{2}{|c|}{ - } & \multicolumn{2}{|c|}{$44: 56$} & \multicolumn{2}{|c|}{$5: 95$} \\
\hline
\end{tabular}

a $\mu \mathrm{mol}$ per mg of dried cell weight.

1) Basal medium in which glucose is replaced by glycerol.

2) Basal medium.

3) Basal medium supplemented with $0.5 \%$ L-arginine.

added to the basal medium, K-582 B was predominantly synthesized, while the addition of L-lysine stimulated K-582 A formation. These results, summarized in Table 2, indicate that addition of lysine may increase the intracellular concentration of lysine and leads to the replacement of Hyarg in position 6 by lysine in the K-582 B molecule. Therefore, the amino acid recognition at position 6 in K-582 A or $\mathrm{B}$ appears to be loosely specific.

\section{Role of Intracellular Amino Acid Pools}

From the results of the preceding experiments, it appears possible that free intracellular amino acids contribute to regulation of K-582 biosynthesis, especially during the idiophase. The concentration and composition of intracellular amino acid pools in three distinct mycelia grown in basal medium, argininesupplemented and glycerol-containig medium, were measured at 4 and 5 days of cultivation (idiophase). Results are summarized in Table 3.

Hyarg, a major constituent of K-582, was greatly influenced. It appeared to be the rate-limiting precursor of K-582. Arginine addition favored Hyarg production and inhibited lysine synthesis. Therefore, K-582 B predominated over K-582 A. Glycerol (1st column, Table 3) completely abolished K-582 production; Hyarg could not be detected in the intracellular pool. Therefore, since the other five constituent amino acids of the antibiotic were synthesized normally, the inhibition of K-582 production by glycerol may be attributed to Hyarg biosynthesis inhibition. This suggests that Hyarg is synthesized from arginine and incorporated into the K-582 molecule.

\section{Effect of Glycerol and Arginine on the Accumulation of Hyarg}

Further investigation on the accumulation of Hyarg which appears to be the rate-limiting amino acid for K-582 biosynthesis, was carried out.

When L-arginine was added in excess to the basal medium, free Hyarg accumulated not only intracel- 
Table 4. Effect of glycerol and arginine on intracellular and extracellular accumulation of Hyarg.

\begin{tabular}{|c|c|c|c|c|c|c|}
\hline \multirow{3}{*}{ Medium $^{a}$} & \multicolumn{4}{|c|}{ Hyarg $(\mu \mathrm{g} / \mathrm{ml})$} & \multirow{2}{*}{\multicolumn{2}{|c|}{ Growth $(\mathrm{mg} / \mathrm{ml})$}} \\
\hline & \multicolumn{2}{|c|}{ Extracellular } & \multicolumn{2}{|c|}{ Intracellular } & & \\
\hline & 5 days & 6 days & 5 days & 6 days & 5 days & 6 days \\
\hline Glucose & trace & trace & 61.71 & 60.04 & 15.80 & 16.20 \\
\hline Glucose + arginine & 72.85 & trace & 137.89 & 192.81 & 19.40 & 20.60 \\
\hline Glycerol & 0 & 0 & 0 & 0 & 16.80 & 18.70 \\
\hline Glycerol + arginine & 0 & 0 & 0 & 0 & 16.10 & 22.80 \\
\hline
\end{tabular}

a Cells were grown in the basal medium containing glucose or glycerol $3 \%$; L-arginine $0.5 \%$ was added when indicated.

lularly but also extracellularly. The amount of intracellular Hyarg in the mycelium grown in the presence of L-arginine was 2 to 3 fold higher than that of mycelium grown in the absence of L-arginine.

However, Hyarg was not produced when glycerol was the sole carbon source in spite of a high arginine concentration. Mycelial growth was abundant under both conditions (with or without L-arginine). These results, shown in Table 4, demonstrate that Hyarg is biosynthesized from arginine and that glycerol probably causes
Fig. 2. Time course of intra- and extra-cellular Hyarg accumulation by $M$. anisopliae U-47.

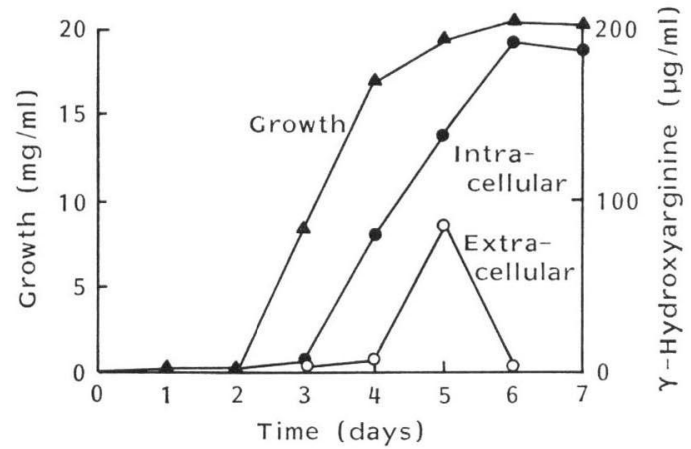
catabolite repression of the enzyme(s) involved in Hyarg biosynthesis.

The time course of extracellular and intracellular accumulation of Hyarg by M. anisopliae U-47 grown in arginine-supplemented medium is shown in Fig. 2. The accumulation of intracellular Hyarg begins at the middle stage of the exponential growth phase and increased steadily thereafter. On the other hand, extracellular Hyarg appears later in the exponential phase, reaches a maximum level on day 5 , then decreases sharply.

\section{Isolation of Hyarg}

M. anisopliae U-47 was cultured in the basal medium supplemented with $0.5 \% \mathrm{~L}$-arginine at $30^{\circ} \mathrm{C}$ for 5 days on a rotary shaker. The cultured broth was filtered on Toyo Roshi No. 2 filter paper. The intracellular amino acids were extracted from the mycelia with boiling water for 15 minutes. The broth filtrate ( 3.5 liters) and the extract (3.5 liters) from the mycelia were combined and passed through a column $(4.5 \times 60 \mathrm{~cm})$ of Amberlite IR-120 ( $\mathrm{H}^{+}$form). After washing with water, the column was eluted with $2.6 \mathrm{M} \mathrm{NH}_{4} \mathrm{OH}$. The eluate containing the amino acid was collected and concentrated in vacuo to remove ammonia. The concentrated solution was applied to a column $(4.0 \times 55 \mathrm{~cm})$ of Dowex $50 \mathrm{WX} 8$ (100 200 mesh) equilibrated with 1 м pyridine - acetate buffer (pH 5.2). The column was eluted with 1.5 liters of the same buffer at a flow rate of $500 \mathrm{ml} /$ hour, then with $2 \mathrm{M}$ pyridine - acetate buffer (pH 5.2). The eluate containing the amino acid was collected and evaporated to dryness under reduced pressure. The dried material was dissolved in a small amount of $2 \mathrm{M}$ pyridine - acetate buffer ( $\mathrm{pH}$ 5.2) and the solution was applied to a column $(4.0 \times 110 \mathrm{~cm})$ of Dowex $50 \mathrm{WX} 8(200 \sim 400 \mathrm{mesh})$ equilibrated with the same buffer. The column was eluted with the same buffer and $15-\mathrm{ml}$ fractions were collected. Frac- 
Fig. 3. IR spectrum $(\mathrm{KBr})$ of threo-L- $\gamma$-hydroxyarginine monoacetate.

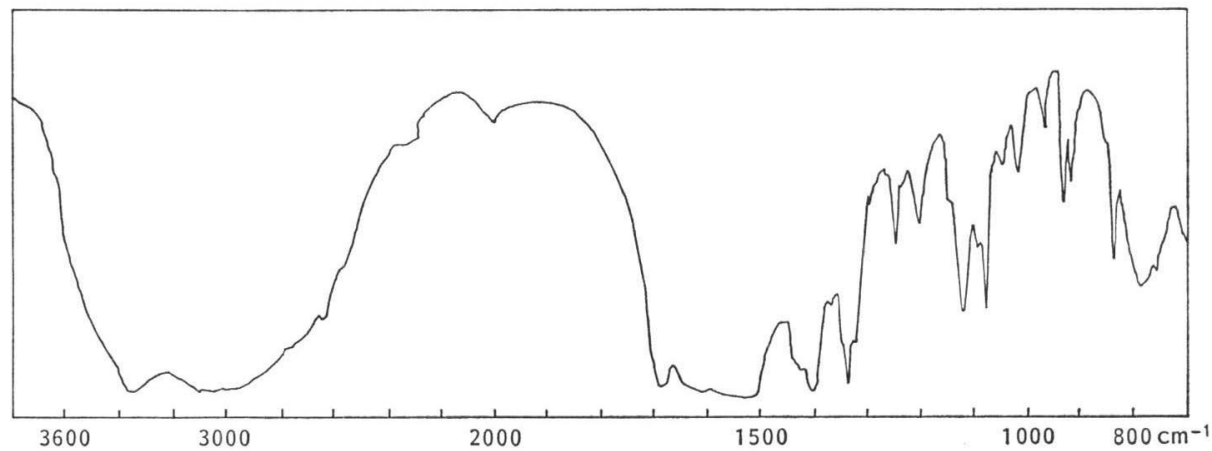

Fig. 4. ${ }^{13} \mathrm{C}$ NMR spectra of threo-I- $\gamma$-hydroxyarginine monoacetate (in $\left.\mathrm{D}_{2} \mathrm{O}\right)$.

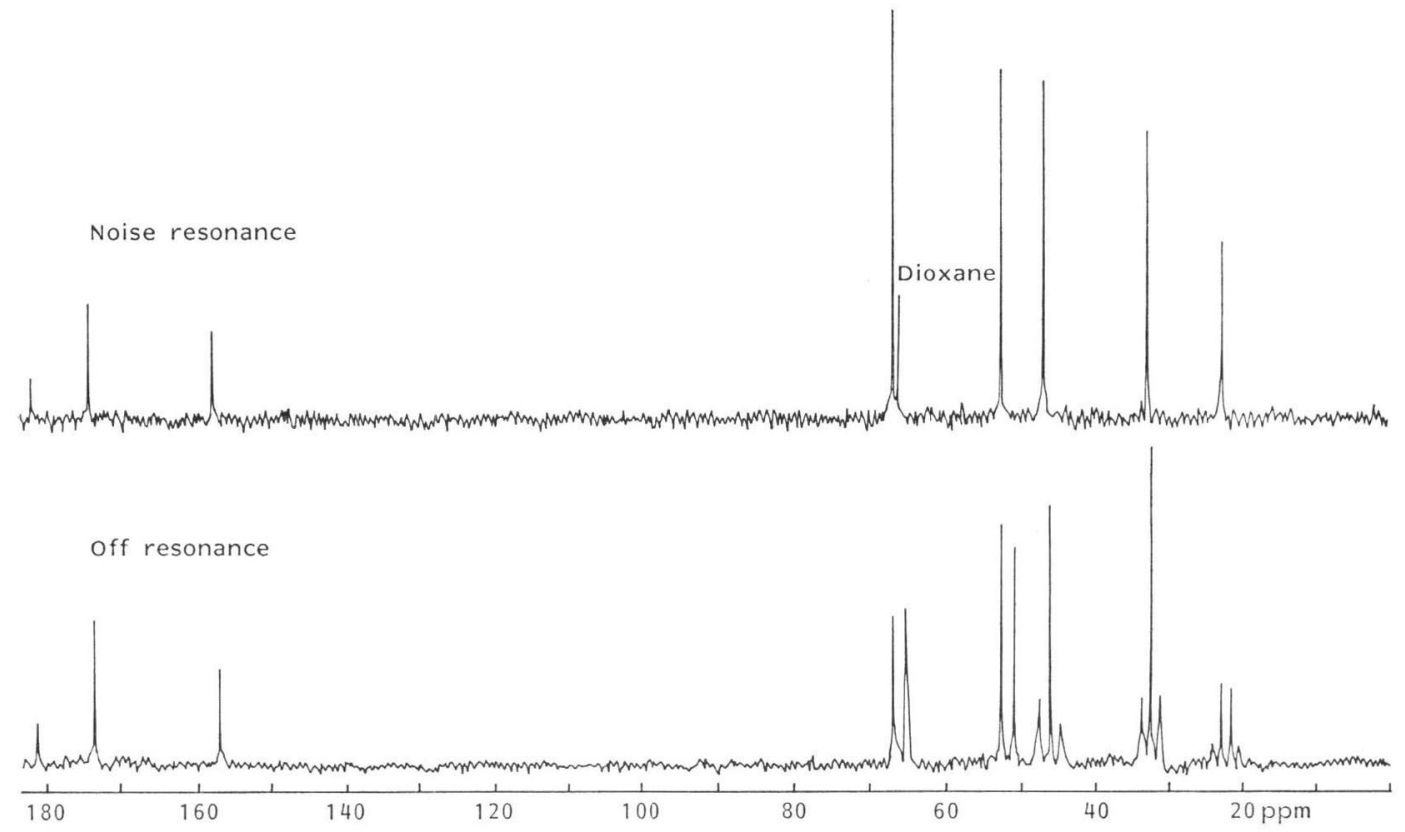

tions 375 to 405, containing only Hyarg were combined and dried in vacuo. The dried material was dissolved in a small amount of water, decolorized with active charcoal and concentrated. By addition of cold ethyl alcohol to the concentrated solution, $800 \mathrm{mg}$ of Hyarg monoacetate was obtained as colorless plate crystals: mp $189 \sim 190^{\circ} \mathrm{C}(\mathrm{dec}) ;[\alpha]_{\mathrm{D}}^{22.5}+11.0^{\circ}(c 1,0.5 \mathrm{M} \mathrm{NaOH})$.

$$
\begin{array}{lll}
\text { Anal Found: } & \mathrm{C} 38.49, \mathrm{H} 7.32, \mathrm{~N} 22.43 . \\
& \text { Calcd for } \mathrm{C}_{8} \mathrm{H}_{13} \mathrm{O}_{5} \mathrm{~N}_{4}: \text { C 38.40, H 7.25, N 22.40. }
\end{array}
$$

No UV absorption maximum was observed. The IR spectrum is shown in Fig. 3. The ${ }^{13} \mathrm{C} \mathrm{NMR}$ spectra are shown in Fig. 4. The compound gave a positive color reaction with ninhydrin and SAKAGUCHI reagents. After treatment with arginase, Hyarg yielded a considerable amount of urea. In the presence of L-amino acid oxidase, the peak corresponding to Hyarg disappeared.

On the basis of the above analytical results and optical rotational data by comparison with MIzUSAKI and MAKISUMI ${ }^{6)}$ data for synthetic threo-L- $\gamma$-hydroxyarginine; $[\alpha]_{\mathrm{D}}^{20}+11.0^{\circ}(c 1,0.5 \mathrm{M} \mathrm{NaOH})$, the isolated amino acid was identified as threo-L- $\gamma$-hydroxyarginine monoacetate. 


\section{Discussion}

The regulatory effects of certain amino acids on peptide antibiotic synthesis have been reported for colistin $^{7,8)}$, actinomycin $\mathrm{D}^{11)}$ and tyrocidine ${ }^{12)}$. In this study, the regulation of $\mathrm{K}-582$ synthesis was ascribed to the amount of endogenous Hyarg. Addition of high amount of L-arginine to the basal medium led to high production of K-582 and significantly determined the proportion of K-582 A and B produced. The exogenously supplied amino acid altered the relative concentration of the amino acid pools; the incorporation of L-lysine or Hyarg into position 6 of K-582 depended on their relative concentration. Moreover, under the same cultural conditions, the Hyarg synthesized was not entirely incorporated into K-582 and accumulated extra- and intra-cellularly.

Hyarg synthesized by the imperfect fungus, $M$. anisopliae, has the threo-configulation. The optical rotation of Hyarg from other natural sources, such as the sea cucumber, $P$. rufescens, and the sea anemone, A. japonica Verrill, is the erythro-isomer ${ }^{8,13)}$.

On the basis of oxygen-18 experiment, Diegelmann et al. ${ }^{14)}$ showed that the transformation of proline into hydroxyproline, which is incorporated into actinomycin D by Streptomyces antibioticus involves molecular oxygen. It is assumed that Hyarg in $M$. anisopliae is biosynthesized from arginine in the participation of molecular oxygen by the same biosynthetic mechanism as that of hydroxyproline in S. antibioticus. The detailed studies on the hydroxylation of arginine in $M$. anisopliae $\mathrm{U}-47$ is in progress using oxygen-18.

Glycerol caused a marked repression of K-582 and Hyarg syntheses. The experimental data obtained under experimental conditions employed in this study suggest catabolite repression of the specific enzyme(s) involved in the biosynthesis of Hyarg.

\section{Acknowledgment}

The authors thank Professor H. OGURA and Dr. K. Furuhata of Kitasato University for their helpful advice on the structural studies of $\gamma$-hydroxyarginine.

\section{References}

1) Kondo, S.; N. Meguriya, H. Mogi, T. Aota, K. Miura, T. Fuji, I. Hayashi, K. Makino, M. Yamamoto \& N. NAKAJIMA: K-582, a new peptide antibiotic. I. J. Antibiotics 33: 533 542, 1980

2) Kondo, S.: New peptide antibiotics and their manufacturing methods. Japan Kokai 54-92,914, July 23, 1979

3) Fujita, Y.: $\gamma$-Hydroxyarginine, a new guanidino compound from a sea cucumber. 1. Isolation and identification. Bull. Chem. Soc. Jpn. 32: 439 442, 1959

4) Makisumi, S.: Guanidino compounds from a sea anemone, Anthopleura japonica Verrill. J. Biochem. 49: $284 \sim 291,1961$

5) Bell, E. A. \& A. S. L. Tirimanna: Occurrence of $\gamma$-hydroxyarginine in plants. Nature 197: 901 902, 1963

6) Mizusaki, K. \& S. Makisumi: Synthesis of four stereoisomers of $\gamma$-hydroxyarginine via the corresponding isomers of $\gamma$-hydroxyornithine. Bull. Chem. Soc. Jpn. 54:470 472, 1981

7) Ito, M.; K. Aida \& T. Uemura: Studies on the bacterial formation of a peptide antibiotic, colistin. III. On the biosynthetic pathway of $\alpha, \gamma$-diaminobutyric acid and relationship between colistin formation and amino acid metabolism in Bacillus colistinus Koyama. Agric. Biol. Chem. 33: 949 958, 1969

8) Ito, M.; K. Aida \& T. Uemura: Formation mechanism of colistin produced by Bacillus colistinus Koyama. Progress in Antimicrob. \& Anticancer Chemother., II. pp. 1128 1137, Univ. Tokyo Press, Tokyo, 1970

9) Schmit, J. C. \& S. Brody: Neurosposa crassa conidial germination; role of endogenous amino acid pools. J. Bacteriol. 124: $232 \sim 242,1975$

10) Wellner, D. \& L. A. Lichtenberg: Assay of amino acid oxidase. Methods in Enzymology. Ed., H. TABOR \& C. W. TABOR, pp. $593 \sim 594$, Academic Press Inc., New York, 1971

11) Katz, E.; C. R. Waldron \& M. L. MelonP: Role of valine and isoleucine as regulators of actinomycin peptide formation by Streptomyces chrysomallus. J. Bacteriol. 82: 600 608, 1961

12) MACH, B. \& E. L. TATUM: Environmental control of amino acid substitutions in the biosynthesis of the antibiotic polypeptide tyrocidine. Proc. Natl. Acad. Sci. U.S. 52: 876 883, 1964

13) FujitA, Y.: $\gamma$-Hydroxyarginine, a new guanidino compound from a sea cucumber. II. Determination of the configuration. Bull. Chem. Soc. Jpn. 33: 1379 1381, 1960

14) Diegelmann, R. F.; O. Ondrejickova \& E. Katz: Oxygen-18 and fluoroproline studies on the synthesis of hydroxyproline and oxoproline in actinomycin. Arch. Biochem. Biophys. 131: 276 287, 1969 